\title{
Redução de sílaba em fala espontânea nas alterações específicas de linguagem $* * *$
}

\author{
Syllable deletion in spontaneous speech of children with specific \\ language impairment
}

\author{
Débora Maria Befi-Lopes* \\ Silmara Rondon**
}

*Fonoaudióloga. Livre Docente em Fonoaudiologia pela Faculdade de Medicina da Universidade de São Paulo (FMUSP). Professora Associada do Curso de Fonoaudiologia da FMUSP. Endereço para correspondência: Rua Cipotânea, 51 São Paulo - SP - CEP 05360-000 (dmblopes@usp.br).

**Fonoaudióloga. Mestranda do Programa de Pós-Graduação em Ciências da Reabilitação do Departamento de Fisioterapia, Fonoaudiologia e Terapia Ocupacional da FMUSP.

***Trabalho Realizado no Laboratório de Investigação Fonoaudiológica em Desenvolvimento da Linguagem e suas Alterações do Departamento de Fisioterapia, Fonoaudiologia e Terapia Ocupacional da FMUSP.

Artigo Original de Pesquisa

Artigo Submetido a Avaliação por Pares

Conflito de Interesse: não

Recebido em 19.02.2009.

Revisado em 30.03.2010, 15.06.2010. Aceito para Publicação em 18.08.2010.

\begin{abstract}
Background: syllable deletion (SD) frequently occurs in Specific Language Impairment and can indicate a deviant factor in the phonologic acquisition process of these subjects. Aim: to verify the occurrence of SD in the spontaneous speech of children with SLI and to verify the influence of word extension and syllable stress in this process. Methods: participants were 27 children with SLI, aged between 3:0 and 5:11 years, in a weekly speech treatment, who presented $50 \%$ of correct answers in specific phonology assessment tasks or who presented speech intelligibility within levels that allowed assessment through spontaneous speech. Speech samples were obtained during a play interaction situation between the researcher and the child and through speech elicited by the presentation of a picture. The occurrence of SD was analyzed considering the following parameters: extension of the produced words, preference for stressed or unstressed syllables, position of the syllable within the word were SD occurred. Results: there was a preference for the production of dissyllabic words $(\mathrm{X} 2=72,49 ; \mathrm{p}<0,001)$; the occurrence of SD was significantly higher in polysyllabic words $(X 2=11,22 ; \mathrm{p}<0,004)$ and on initial syllables $(X 2=34,99$; $\mathrm{p}<0,001)$. Unstressed syllables were more often reduced $(\mathrm{Z}=-5.79, \mathrm{p}<0001)$. Conclusion: the preference for the production of dissyllabic words reassures the difficulty of these children with complex syllabic structures and, in part, explains their spontaneous speech unintelligibility. The predominance of unstressed syllable deletion indicates the preference for producing the nucleus of words, where emphasis is given to the stressed syllable during language expression.
\end{abstract}

Key Words: Child; Language; Evaluation.

\section{Resumo}

Tema: a redução de sílaba (RS) ocorre frequentemente nas Alterações Específicas de Linguagem (AEL), podendo indicar um fator desviante no processo de aquisição fonológica destes sujeitos. Objetivo: verificar a ocorrência de RS na fala espontânea de crianças com AEL e a influência dos fatores extensão das palavras e tonicidade para sua ocorrência. Método: foram sujeitos 27 crianças com AEL, com idades entre 3:0 e 5:11 anos, em tratamento fonoaudiológico semanal, que apresentaram 50\% de acertos em provas específicas de fonologia realizadas ou que apresentaram inteligibilidade de fala passível de análise pela fala espontânea. As amostras de fala foram obtidas a partir de interação lúdica com a pesquisadora e pelo discurso eliciado por figuras. A ocorrência de RS foi analisada considerando-se: extensão das palavras produzidas, preferência por sílabas tônicas ou átonas e posição das sílabas nas palavras em que ocorreu RS. Resultados: houve predomínio na produção de palavras dissílabas $\left(X_{2}=72,49 ; p<0,001\right)$, a ocorrência de redução de sílaba foi significantemente maior nas palavras polissílabas $\left(X_{2}=11,22 ; p<0,004\right)$ e as sílabas iniciais foram mais reduzidas $\left(\mathrm{X}_{2}=34,99 ; \mathrm{p}<0,001\right)$. As sílabas átonas foram reduzidas com maior frequência $(Z=-5,79 ; p<0,001)$. Conclusão: a preferência pela produção de palavras dissílabas confirma a dificuldade dos sujeitos com estruturas silábicas complexas e justifica parte de sua ininteligibilidade em fala espontânea. A predominância da redução de sílabas átonas indica a preferência pela produção do núcleo das palavras, em que a ênfase é dada na sílaba tônica durante a expressão da linguagem.

Palavras-Chave: Criança; Linguagem; Avaliação.

Referenciar este material como: 


\section{Introdução}

As Alterações Específicas da Linguagem (AEL) têm ocorrência significativa dentre os quadros que acometem o processo normal de aquisição e desenvolvimento da linguagem, sendo a fonologia um dos subsistemas da linguagem mais comprometidos $^{1-4}$.

Durante a aquisição fonológica é muito importante a habilidade de segmentação de palavras ouvidas, que se dá, sobretudo, por meio da percepção das características sonoras dos fonemas, inclusive no que se refere ao acento silábico e à posição das sílabas nas palavras, aspectos estudados pela fonologia métrica ${ }^{5-7}$.

A redução ${ }^{8}(\mathrm{RS})$ ou apagamento ${ }^{9}$ de sílaba, considerada um processo fonológico do desenvolvimento, caracterizado pela eliminação de uma sílaba ou mais sílabas durante a produção de uma palavra, que é de grande frequência nas AEL, ocorre em maior número durante o desenvolvimento típico em sílabas átonas, existindo também a influência da extensão das palavras para sua ocorrência ${ }^{10-11}$.

Ademais, durante o desenvolvimento típico a ocorrência da redução de sílaba é maior em posição medial e, em casos de AEL, ocorre em mais de uma sílaba em uma mesma palavra. Esse fato pode indicar um fator desviante no processo de aquisição fonológica ${ }^{12}$, devendo ser considerado pelo fonoaudiólogo durante a realização de diagnóstico diferencial nos casos de AEL.

Assim, os objetivos dessa pesquisa foram verificar a ocorrência do processo fonológico de redução de sílaba em crianças com AEL em situação de fala espontânea e a influência dos fatores extensão das palavras e tonicidade para sua ocorrência.

\section{Método}

Esta pesquisa foi desenvolvida no Laboratório de Investigação Fonoaudiológica em Desenvolvimento da Linguagem e suas Alterações do Departamento de Fisioterapia, Fonoaudiologia e Terapia Ocupacional da Faculdade de Medicina da Universidade de São Paulo (FMUSP). Houve aprovação da Comissão de Ética em Pesquisa do HC-FMUSP tanto no que se refere ao seu teor bem como aos aspectos constantes do Termo de Consentimento Livre e Esclarecido assinado pelos responsáveis pelas crianças que foram estudadas (protocolo de autorização número 535/06). O projeto foi financiado pela Fundação de Amparo à Pesquisa de São Paulo - Fapesp (processo número 2006/ 61458-0).
Foram sujeitos desta pesquisa 27 crianças que procuraram atendimento fonoaudiológico no Laboratório de Investigação Fonoaudiológica em Desenvolvimento da Linguagem e suas Alterações do Curso de Fonoaudiologia da FMUSP, ou que estiveram em processo terapêutico semanal durante a vigência da execução da coleta de dados, os quais foram incluídos obedecendo aos seguintes critérios:

- autorização dos pais e/ou responsáveis para participação na pesquisa;

. respeitar os critérios de exclusão para diagnóstico de AEL, a saber: perdas auditivas, lesões cerebrais focais ou disfunções neurológicas adquiridas, comprometimento motor do desenvolvimento de fala, distúrbios abrangentes do desenvolvimento ou síndromes ${ }^{13-14}$, tendo como fator de inclusão, o baixo desempenho em testes padronizados de linguagem ${ }^{15}$. Para o diagnóstico foi utilizado o $\mathrm{ABFW}^{16}$, Provas de Utilização e Compreensão de Preposições ${ }^{17}$, Compreensão de $\operatorname{Adjetivos}{ }^{18}$ e Vocabulário Receptivo ${ }^{19}$;

. faixa etária entre 3:0 e 5:11 anos, de ambos os sexos (sendo 23 crianças do gênero masculino e 4 do gênero feminino) com desempenho abaixo do esperado em pelo menos três das provas de linguagem aplicadas, além das alterações na prova de fonologia ${ }^{8}$;

. conseguir realizar, pelo menos $50 \%$ das provas de imitação de palavras e nomeação de figuras da prova de fonologia do $\mathrm{ABFW}{ }^{16}$;

- possuir, no mínimo, oralidade suficiente analisável para a realização em prova de discurso e na prova de pragmática ${ }^{20}$, que foram as amostras analisadas para coleta da fonologia em fala espontânea. Sujeitos cujas produções orais não permitiram transcrição fonológica, nas provas referidas, não puderam compor o grupo aqui estudado, pois nosso objetivo foi descrever as características fonológicas da primeira amostra analisável de fala espontânea.

Para a realização do presente estudo foram necessários:

. um gravador digital com cabo USB para gravação fidedigna das emissões das crianças nas provas de imitação e nomeação;

. um microfone de lapela acoplável ao gravador; - brinquedos para interação lúdica com as crianças a fim de coletar fala espontânea (Prova de Pragmática ${ }^{20}$ )

. figura para coleta do discurso (aqui foi utilizada uma figura representando uma festa de aniversário ${ }^{21}$ - Eva Furnari, ou a figura para eliciação de discurso 
do ABFW - teste de linguagem infantil ${ }^{16}$ e utilizamos na transcrição a amostra de fala que apresentou o maior número de palavras inteligíveis passíveis de análise);

. uma filmadora digital, pois na maioria dos casos, só é possível a transcrição da fala espontânea a partir da observação da situação, ou seja, do contexto comunicativo, uma vez que a ininteligibilidade de fala pode impedir a observação de se aquela emissão refere-se, ou não, à temática da interação; . um notebook, Pentium 4.

Após a seleção dos sujeitos como já descrito na casuística deste mesmo item do presente artigo, foi realizada a coleta de dados como segue:

. todas as crianças que se caracterizaram como sujeitos da pesquisa foram primeiramente avaliadas através de provas específicas de avaliação da fonologia - nomeação de figuras e imitação de palavras $^{8}$, em situação dirigida, dentro da sala de terapia que estão acostumadas a frequentar. Para evitar possível cansaço na realização das provas, estas foram aplicadas em dias separados (com diferença de sete a quinze dias entre uma avaliação e outra, sendo que $50 \%$ das crianças realizaram primeiro a prova de imitação e $50 \%$ a prova de nomeação). Tal procedimento foi adotado para garantir que a fala dos sujeitos era analisável, uma vez que a ininteligibilidade de fala de muitas das crianças com AEL não permite transcrição.

TABELA 1. Estatística descritiva do total de palavras de acordo com a extensão.

\begin{tabular}{c|c|c|c|c|c}
\hline & Média & Mediana & DP & Mínimo & Máximo \\
\hline dissílabo & 14,50 & 10,50 & 14,33 & 0 & 58,00 \\
trissílabo & 7,37 & 5,00 & 5,72 & 0 & 21,00 \\
polissílabo & 1,48 & 1,00 & 1,66 & 0 & 6,00 \\
\hline
\end{tabular}

TABELA 2. Ocorrência de RS de acordo com a extensão.

\begin{tabular}{c|c|c|c|c}
\hline & Mediana & $\begin{array}{c}\text { Mediana do Número } \\
\text { de Ordem }\end{array}$ & $\mathrm{X}_{2}$ & $\mathrm{p}$-valor \\
\hline dissílabos & 0 & 1,61 & & $0,004^{*}$ \\
trissílabos & 0,46 & 1,98 & 11,22 & $(\mathrm{di}<\mathrm{tri}<$ poli $)$ \\
polissílabos & 0,50 & 2,41 & & \\
\hline
\end{tabular}

Após a aplicação destas provas, todos os sujeitos que conseguiram realizar pelo menos $50 \%$ do total de cada uma das provas, foram gravados (em outro dia), em situação de interação lúdica com a pesquisadora (conforme orientado por Fernande ${ }^{20}$ ) e coleta da fala espontânea a partir de figura (discurso), a fim de se realizar o levantamento para posterior análise fonológica, seguindo os mesmos parâmetros, a saber:

- produção de palavras com o processo fonológico redução de sílaba (conforme descrito por Wertzner $^{8}$ ) observando se há preferência, ou não, pela utilização das tônicas ou átonas;

- posição na palavra da(s) sílaba(s) reduzida(s) quando da ocorrência de redução de sílabas.

Após coleta e transcrição dos dados, estes foram tabulados respeitando-se análise proposta no item procedimento do presente projeto e passaram por tratamento estatístico cabível para respostas aos objetivos preestabelecidos.

\section{Resultados}

Para a análise estatística dos dados foram utilizados os seguintes testes não paramétricos: Friedman, McNemar e Wilcoxon. O nível de significância adotado foi de 5\%. Os resultados estatisticamente significantes estão e negrito.

A Tabela 1 apresenta a ocorrência de palavras de acordo com o número de sílabas, sendo que o teste Qui-quadrado mostrou que o predomínio de palavras dissilábicas no repertório das crianças analisadas é estatisticamente significante, independente da prova realizada, quando comparado ao número de palavras trissilábicas seguido do número de palavras polissilábicas $\left(\mathrm{X}_{2}=72,49 ; \mathrm{p}<0,001 *\right)$.

A fim de verificar se há efeito de extensão, foi calculada a proporção de RS de acordo com o tipo de palavra produzida, visto que as crianças produzem significativamente mais palavras dissilábicas e menos palavras polissilábicas.

Na Tabela 2, pode-se observar que a partir do cálculo de proporção fica evidenciado que a ocorrência de RS é influenciada pela extensão da palavra, dado que o número de ocorrências de RS em palavras polissilábicas é maior, quando comparado ao número de ocorrências do referido processo em palavras trissilábicas e em seguida às palavras dissilábicas. O teste Qui-quadrado mostrou que essa diferença é estatisticamente significante. 
Os resultados da análise da ocorrência de RS de acordo com a posição na palavra, mostraram predomínio estatisticamente significante de RS em sílaba inicial, quando comparado à ocorrência do mesmo processo em sílabas mediais e finais $\left(\mathrm{X}_{2}=34,99 ; \mathrm{p}<0,001 *\right)$. Esses dados também foram encontrados pela análise estatística descritiva (RS em sílaba inicial: média $=2,15$; mediana =1,5; $\mathrm{DP}=2,34 ;$ mínimo=0; máximo=9).

Ademais, na Tabela 3 podem ser observados os resultados da análise da ocorrência de RS de acordo com a tonicidade da sílaba, predominando significativamente em sílabas átonas, para todas as posições de sílabas nas palavras produzidas pelos sujeitos da pesquisa.

\section{Discussão}

A partir dos dados encontrados no presente trabalho, foi possível destacar algumas considerações acerca do desenvolvimento fonológico de crianças com Alterações Específicas de Linguagem (AEL).

O processo fonológico de RS é caracterizado como um processo em que se omite uma ou mais sílabas de uma palavra. Sua ocorrência é esperada durante fases iniciais da aquisição e desenvolvimento fonológico, sendo que costuma ser superado por volta dos 2:6 anos ${ }^{8}$.

A ocorrência desse processo fonológico compromete a inteligibilidade de fala das crianças com AEL e pode estar ligada à ausência ou diminuição de habilidades relacionadas à segmentação de palavras em sílabas, percepção das características acústicas e prosódicas das palavras, organização dos fonemas da língua e dificuldades em linguagem expressiva. Ademais, a ocorrência de RS pode sofrer influência da extensão das palavras ${ }^{5-7,22}$.

A partir da análise dos resultados obtidos foi possível identificar que os sujeitos da pesquisa produziram número maior e estatisticamente significativo de palavras dissilábicas em seu repertório durante as duas situações de fala espontânea propostas, quando comparado ao número de palavras trissilábicas e polissilábicas (dissílabos > trissílabos > polissílabos). Esse dado é contrário ao que foi encontrado por $\mathrm{Galea}^{23}$, em estudo com crianças em desenvolvimento típico com faixas estarias entre 2:1 e 3:0 anos em que, na situação de fala espontânea, em que as crianças produziram maior número de palavras polissilábicas. Esses dados podem indicar uma imaturidade no sistema fonológico das crianças com AEL estudadas nesta pesquisa, uma vez que têm desempenho pior do que o de crianças de faixas etárias inferiores, as quais se encontram em desenvolvimento típico.
TABELA 3. Ocorrência de RS de acordo com a tonicidade.

\begin{tabular}{lccccccc}
\hline & Média & Mediana & DP & Mínimo & Máximo \\
\hline tônica & 0,04 & 0 & 0,19 & 0 & 1,00 \\
átona & 3,43 & 3,00 & 3,46 & 0 & 15,00 \\
& \multicolumn{6}{c}{$\mathrm{Z}=-5,79 ;$ p-valor $<0,001^{*}$} \\
\hline
\end{tabular}

Ademais, foram analisadas as ocorrências de RS de acordo com a posição das sílabas reduzidas nas palavras produzidas pelos sujeitos, em que os resultados indicaram um número maior de RS em sílabas encontradas na posição inicial das palavras, quando comparado ao número de ocorrências do mesmo processo em sílabas mediais e finais. No desenvolvimento típico as sílabas mediais costumam ser as mais reduzidas, o que mostra um padrão alterado do processo de aquisição e desenvolvimento fonológico nas crianças com $\mathrm{AEL}^{12}$.

Além disso, verificou-se nesta pesquisa, a partir dos resultados obtidos, que as crianças com AEL tendem a reduzir as sílabas átonas das palavras. Esse dado corrobora com os dados da literatura para esta população, os quais são encontrados tanto para falantes do Português como para falantes de línguas como o Inglês, o Catalão, o Francês e o Italiano $^{12-13,24-25}$. Ademais, a estrita relação com o padrão trocaico de distribuição das sílabas nas palavras, como ocorre no Português Brasileiro, pode influenciar na ocorrência de redução de sílabas átonas ${ }^{13}$. Há ainda evidências de que a redução de sílabas átonas possa ser um marcador das $\mathrm{AEL}^{25}$.

Crianças com AEL apresentam grandes dificuldades em produzir e manter nas palavras as sílabas átonas não-finais. Essa dificuldade pode refletir em alterações gramaticais nas estruturas sintáticas de palavras e sentenças, uma vez que artigos e preposições se apresentam como palavras monossilábicas átonas e podem ser omitidas, prejudicando a inteligibilidade de fala ${ }^{13,25}$.

Essa preferência pela produção das sílabas tônicas pode ser explicada com base na fonologia métrica, como sendo ocasionada pelo aumento da saliência acústica (acento silábico) em relação às sílabas átonas, o que facilita a percepção e a organização dos fonemas para a sua produção nas palavras $^{5-7,24}$.

Crianças em desenvolvimento típico conseguem perceber e distinguir diferenças relacionadas a acento silábico e extensão das palavras muito precocemente, já em seu primeiro ano de vida ${ }^{26}$. Crianças com AEL costumam apresentar um déficit 
perceptual que afeta o uso da informação fonológica na memória de trabalho, o que os conduz a dificuldades de compreensão e expressão da linguagem relacionadas à fonologia e à sintaxe. Dessa maneira, como foi demonstrado no presente estudo, esses sujeitos apresentam maiores dificuldades em segmentar palavras em sílabas e reconhecer suas características fonológicas e prosódicas, a fim de organizá-las na linguagem expressiva $^{3,26}$.

Os aspectos de extensão das palavras e acento silábico podem estar intimamente relacionados ao desempenho semântico e fonológico das crianças com $\mathrm{AEL}^{26}$.

Assim, entende-se que o desenvolvimento de habilidades fonológicas é de extrema importância para o desenvolvimento dos demais subsistemas da linguagem. Além disso, tarefas mais complexas relacionadas à aquisição de conceitos e à elaboração de idéias requerem maiores e também mais complexas habilidades fonológicas para serem realizadas com sucesso.

\section{Conclusão}

Os dados encontrados nesta pesquisa mostraram que crianças com AEL têm preferência pela produção de palavras dissilábicas, o que não acontece durante o desenvolvimento típico no qual, em situação de fala espontânea, é maior a ocorrência de palavras polissilábicas. Essa restrição de vocabulário pode se apresentar tanto como um déficit semântico característico dessas alterações quanto como reflexo do comprometimento fonológico desses sujeitos para representação fonológica do item lexical, o que prejudica seu desempenho em linguagem expressiva, conforme é discutido na literatura.

Ademais, foi possível constatar que a ocorrência de RS é influenciada pela extensão da palavra, dado que o número de ocorrências de RS em palavras polissilábicas é maior, quando comparado ao número de ocorrências do referido processo em palavras trissilábicas e em seguida às palavras dissilábicas.

Além disso, os resultados aqui apresentados encontram-se em concordância com a literatura no que se refere à realização de RS e sua relação com a posição das sílabas nas palavras e o acento silábico. Os sujeitos desta pesquisa realizaram de maneira significativa o processo fonológico RS em sílabas átonas e em posição inicial nas palavras. Esse tem se mostrado um possível marcador das AEL, como descrito na literatura e compromete a inteligibilidade de fala desses indivíduos.

Essas dificuldades relacionadas à fonologia podem prejudicar o desenvolvimento da linguagem em geral, afetando um ou mais de seus subsistemas. Por isso é importante avaliar e entender cada um desses subsistemas separadamente, considerando a importância da influência do desenvolvimento fonológico para o sucesso tanto na compreensão como na expressão da linguagem.

\section{Referências Bibliográficas}

1. Wagner CR, NettelbladT B, Sahlen B, Nilholm C. Conversation versus Narration in pre-school children with Language Impairment. Int J Commun Dis. 2000 JanMar;35(1):83-93.

2. Orsolini $\mathrm{M}$ et al. Nature of Phonological delay in children with SLI. Int J Lang Commun Dis. ISSN: 1368-2822. 2001 Jan-Mar;36(1):63-90.

3. Joanisse MF, Seidenberg MS. Phonology and Syntax in Specific Language Impairment: Evidence from a connectionist model. Brain Lang. ISSN: 0093-934X. 2003 Jul;86(1):40-56.

4. Newbury DF, Bishop DVM, Monaco AP. Genetic influences in Language Impairment and Phonological shortterm memory. Trends Cogn Sci. ISSN: 1364-6613. 2005 Set;9(11): 528-34.
5. Gerken L. A metrical template account of children's weak sillabe omission from multisyllabic words. J Child Lang. ISSN: 0305-0009. 1994;21(3):565-84.

6. Turk AE, Jusczyk PW, Gerken L. Do English learning infants use syllabe weight to determine stress? Lang Speech. ISSN: 0023-8309. 1995;38:143-58.

7. Jusczyk PW, Houston DM, Newsome M. The beginnings of word segmentation in english-learning infants. Cogn Psychol. ISSN: 0010-0285. 1999;35(1):45-59.

8. Wertzner HF. Fonologia. In: Andrade CRF, Befi-Lopes DM, Fernandes FDM, Wertzner HF. ABFW - teste de linguagem infantil nas áreas de fonologia, vocabulário, fluência e pragmática. $2^{\mathrm{a}}$ ed. Carapicuiba: Pró-Fono; 2004b. p. 5-32. 
9. Maldonade IR, Mota IR. Vocalic lengthening and medial coda omission in phonological disorders. Pró-Fono Rev Atual Cient. 2010 Jan-Mar;22(1):61-6.

10. Dodd B, Holm A, Hua Z, Crosbie S. Phonological development: a normative study of British English-speaking chidren. Clin Linguist Phon. ISSN: 0269-9206. $2003 ; 17(8): 617-43$.

11. James D. Does the number of syllabes in words affect weak syllabe deletion in tipically developing children aged 3 to 7 years speaking Australian English. In: World Congress of the International Association of Logopedics and Phoniatrics (IALP), 27, 2007. Copenhagem: Dinamarca. International Association of Logopedics and Phoniatrics (IALP), 2007. p. 69.

12. Aguilar-Mediavilla EM, Sanz-Torrent M, Serra-Ravento MA. Comparative study of the phonological of pre-school children with specific language impairment (SLI), language delay (LD) and normal acquisition. Clin Linguist Phon. ISSN: 0269-9206. 2002;16(8):573-96.

13. Bortollini U, Leonard L. Phonology and Children with SLI - Status of structural constraints in two languages. J Commun Dis. ISSN: 0021-9924. 2000;33(2):131-50.

14. Befi-Lopes DM. In: Ferreira LP, Befi-Lopes DM, Limongi SCO. Tratado de Fonoaudiologia. São Paulo: Roca; 2004. p. 987 - 1000.

15. Befi-Lopes DM, Palmieri TM. Análise dos Processos Fonológicos utilizados por crianças com alteração no desenvolvimento da linguagem. J Bras Fonoaudiol. 2000 Jul-Set;4: 48-58.

16. Andrade CRF, Befi-Lopes DM, Fernandes FDM, Wertzner HF. ABFW: Teste de linguagem infantil nas áreas de fonologia, vocabulário, fluência e pragmática. $2^{\mathrm{a}}$ ed. Barueri (SP): Pró-Fono; 2004.

17. Puglisi ML, Befi-Lopes DM, Takiuchi, N. Utilização e compreensão de preposições por crianças com Distúrbio Específico de Linguagem. Pró-Fono Rev Atual Cient. 2005;17( 3):331-44.
18. Befi-Lopes DM, Almeida LMS, Takiuchi $\mathrm{N}$. Compreensão de adjetivos em crianças com distúrbio específico de linguagem. Rev Soc Bras Fonoaudiol. 2005; $10(1): 18-28$.

19. Morselli AA. Identificação de Figuras: comparação entre crianças em desenvolvimento normal e com alterações no desenvolvimento da linguagem. 2003. [Dissertação]. São Paulo(SP): Faculdade de Filosofia, Letras e Ciências Humanas da Universidade de São Paulo; 2003.

20. Fernandes FDM. Pragmática. In: Andrade CRF, BefiLopes DM, Fernandes FDM, Wertzner HF. ABFW - Teste de Linguagem Infantil nas áreas de fonologia, vocabulário, fluência e pragmática. $2^{\mathrm{a}}$ ed. Barueri (SP): Pró-Fono; 2004.

21.Lahey E; Edwards J. Naming errors of children with specific language impairment. J Speech Hear Disord 1999; 42(1):195-205.

22. Befi-Lopes DM, Gândara JP, Araújo K. Aquisição do sistema fonológico em crianças com alterações no desenvolvimento de linguagem. Pró-Fono Rev Atual Cient. 2003 Jan-Abr;15(1):19-30.

23. Galea DES. Percurso da aquisição dos Encontros Consonantais, Fonemas e Estruturas Silábicas em crianças de 2:1 a 3:0 anos de idade. 2008. 226 f. [tese]. São Paulo(SP): Faculdade de Filosofia, Letras e Ciências Humanas da Universidade de São Paulo; 2008.

24. Befi-Lopes DM, Gândara JP, Araújo K. Padrões silábicos produzidos por crianças com alteração no desenvolvimento de linguagem. Pró-Fono Rev Atual Cient. 2003 JanAbr;15(1): 9-18.

25. Maillart C, Parisse C. Phonological deficits in French speaking children with SLI. Int J Lang Commun Dis. ISSN: 1368-2822. 2006 Mai-Jun;41(3): 253-74.

26. McDonald JL. Language Acquisition: the Acquisition of Linguistic Structure in Normal and Special Populations [Review]. Psychology Annual Reviews. Palo Alto (CA). 1997;48:215-41. 